Technische Infrastruktursysteme

\title{
Verwundbarkeiten durch Sektorkopplung
}

\author{
Im Hinblick auf die Zukunft technischer Infra- \\ strukturen ist neuerdings oft von gekoppelten \\ Systemen die Rede. Durch diese zunehmende \\ Vernetzung werden die Systeme auch anfälliger \\ gegenüber Störungen. \\ Von Jens Libbe und Ulrich Petschow
}

und Materialfluss einer anderen Infrastruktur. Solche Kopplungen stehen in einem zeitlichen wie räumlichen Zusammenhang. Vor allem aber ist es die Digitalisierung, die zu einem verstärkten Kommunikations- und Informationsfluss zwischen Infrastrukturen und damit vielfältig neuen, letztlich auch organisatorischen, Zusammenschlüssen führt.

\section{Optionen für neue Systemlösungen}

Kopplungen zwischen Infrastruktursektoren gibt es seit Langem. Im Zentrum steht dabei der Energiesektor, von dem andere technische Systeme abhängig sind. Auch Kopplungen zwischen Teilsektoren innerhalb eines Sektors sind lange bekannt, beispielsweise die Kopplung zwischen Stromerzeugung, -umwandlung und -verteilung. Neu sind die rasant steigende Abhängigkeit von Strom und Information sowie die zunehmend wechselseitige Abhängigkeit sowohl zwischen Sektoren, Teilsektoren und sektorübergreifenden Teilsektoren. In diesem Zusammenhang wird heute von Sektorkopplung (oder auch Sektorenkopplung) gesprochen und der Begriff meist auf die Bereiche Elektrizität, Wärme/Kälte und Verkehr (vor allem Elektrofahrzeuge) bezogen (Agentur für Erneuerbare Energien 2016; Quaschning 2016).

Insbesondere die dynamische Entwicklung im Bereich der Informations- und Kommunikationstechnologien (IKT) ist es, die nicht nur eine verbesserte Steuerung von technischen Systemen im Sinne der Effizienz ermöglicht, sondern auch zu veränderten physischen und organisatorischen Verknüpfungen zwischen Infrastrukturen führt.

Sektorkopplungen sind die Voraussetzung für erhöhte Effizienzen und neuartige Systemlösungen. So bedarf es beispielsweise heute viel weniger als in der Vergangenheit einer Energieversorgung mit sehr großen Reservekapazitäten auf der Angebotsseite. Stattdessen kann sowohl die Einspeisung und Speicherung von Energie als auch der Verbrauch bedarfsgerecht über intelligente Netze („Smart Grids“) geregelt werden. Auf diese Weise kann Volatilität erneuerbarer Energien aufgefangen und die Widerstandsfähigkeit von Energieinfrastrukturen erhöht werden, da die Versorgung nicht von einer einzigen Versorgungsanlage oder einem einzelnen Versorgungssystem abhängig ist. Die Kopplung und damit die Kombination verschiedener Teilsysteme gewährleistet, dass auf Ausfälle besser reagiert werden kann.

Das Beispiel zeigt, dass Informations- und Kommunikationstechnologien nicht nur der unmittelbaren technischen Systemsteuerung dienen, sondern auch helfen, vergleichsweise 
heterogene und teilautonome Teilsysteme besser aufeinander abzustimmen. Dies zeigt sich auch im Bereich der Mobilität, in der es heute darum geht, individuelle und kollektive Fortbewegungsformen so miteinander interagieren zu lassen, dass ein Wechsel zwischen den Teilsystemen (etwa Bahn, ÖPNV, Fahrrad, PKW) problemlos möglich ist.

Die zunehmende Bedeutung von infrastrukturellen Kopplungen hat viel mit den elementaren Charakteristika von Infrastrukturen zu tun. Über die Systeme werden Grunddienstleistungen für die Allgemeinheit erbracht. Strom, Wärme, Daten und so weiter sollen universell, kontinuierlich und an jedem Ort zur Verfügung stehen. Damit diese Versorgungssicherheit auch in Zukunft gewährleistet ist, müssen Systeme so aufgebaut sein, dass eine Unterbrechung der Leistungsbereitstellung nahezu ausgeschlossen ist. Dies wird gewährleistet, indem Erzeugungsanlagen redundant, also mit ausreichender Reservekapazität, geplant, mit anderen Anlagen vernetzt und mit ausreichend Speicherkapazität verbunden werden. Kopplungen sorgen also gleichermaßen für stabile Versorgung auch dann, wenn beispielsweise einzelne erneuerbare Energien aufgrund unterschiedlicher Witterungsbedingungen nicht ständig zur Verfügung stehen.

Infrastrukturkopplungen führen auch zu Anpassungen der infrastrukturellen Mengengerüste. Der großräumigen Übertragung etwa von Strom auf Hochspannungsnetzebene stehen Tendenzen einer kleinräumigen „zellulären“ Versorgungsphilosophie gegenüber, bei der abhängig von lokalen Gegebenheiten quasi autonome Versorgungsstrukturen geschaffen werden. Das heißt, an die Stelle großer gekoppelter Verbunde treten kleinräumige Verknüpfungen in lokalen Netzen („Micro Smart Grid“) auf der Ebene von Ortsteilen, Quartieren oder gar Gebäuden. Lernende Algorithmen sorgen dabei für die notwendige Stabilität des dezentralen Systems. Damit ist eine größere Vielfalt an möglichen Kopplungen verbunden, was Fragen sowohl im Hinblick auf die geeignete Maßstabsebene von Kopplungen als auch im Hinblick auf eine flächendeckende Versorgungssicherheit aufwirft.

\section{Anpassungen von Sicherheitsphilosophien}

Die zunehmenden Abhängigkeiten zwischen Infrastrukturen werden insbesondere in den Diskursen zu den sogenannten kritischen Infrastrukturen thematisiert. Rinaldi et al. (2001) haben die Interdependenzen zwischen (zwei) Infrastruktursystemen anhand von sechs Dimensionen differenziert: Charakteristika der Infrastrukturen, Betriebszustand, Typen der Interdependenzen, Umgebungsbedingungen, Kopplung und Reaktionsverhalten und schließlich Fehlertyp. Bereits diese eher einfache abstrakte Differenzierung verdeutlicht, dass vielfältige Kopplungs- und Rückkopplungseffekte möglich sind. Die Kopplungen mehrere Infrastruktursysteme führt zu der Herausbildung von "System of Systems“, welche als komplexe Systeme begriffen werden. Tondel et al. (2017) analysieren den gegenwärtigen Forschungsstand und stellen fest, dass es selbst bei alleiniger Betrachtung der Interdependenz von Energie-und IKT-Systemen bereits an geeigneten Methoden für die Identifikation der vielfältigen Interdependenzen mangele.

\section{Strategien im Umgang mit Risiken}

Schaut man sich Extremereignisse aus der jüngeren Zeit mit Blick auf die Auswirkungen auf Infrastrukturen oder besser die Bereitstellung von Infrastrukturdienstleistungen an, ist festzustellen, dass Extremereignisse zu erheblichen Beeinträchtigungen führen können. Diese sind aber in der Regel räumlich eingegrenzt und Kaskadeneffekte stellen eher die Ausnahme dar. Dazu trägt bei, dass nicht zuletzt in den Kommunen systematisch Ursachenanalysen vorgenommen werden und diese in Infrastrukturplanungen einfließen.

Kaskadeneffekte können ausgelöst werden insbesondere durch Wetterextremereignisse oder - ein Thema das immer bedeutsamer wird - durch Cyberattacken in Stromnetze. Die Auswirkungen können potenziell weitreichend sein. In Europa haben unter anderem zwei Ereignisse Aufmerksamkeit erregt. In Italien kam es im Jahre 2003 zu einem landesweiten Blackout. Auslöser war ein unwetterbedingter Kurzschluss in der Schweiz, der zu einem lokalen Stromausfall führte. Die Stromlieferungen von der Schweiz nach Italien fielen, aus Gründen der Überlastung, aus und der an die Stelle tretende Import aus Frankreich führte zu einer weitergehenden Überlastung der Netze. In Italien kam es zu einer Einschränkung der Stromversorgung und zugleich wurden die IKT-Netze mit beeinträchtigt. Ein gegenseitiges Aufschaukeln (Strom und IKT) führte in der Folge zu einem vollständigen Blackout. Der Ausfall konnte relativ schnell behoben werden, allerdings waren begünstigende Konstellationen vorhanden, etwa eine schwache Energienachfrage am Wochenende (Buldyrev et al. 2010). Im November 2005 kam es, im Kontext einer Überführung eines Kreuzfahrtschiffes auf der Ems, durch einen Kurzschluss zu europaweiten Kaskadeneffekten im Stromnetz und damit Stromausfällen (Bundesnetzagentur 2017). Auslöser war dabei, dass es unterlassen wurde, vor der Überführung des Schiffes zwei Hochspannungsleitungen abzuschalten.

Festzuhalten ist, dass in der Praxis weitreichende Infrastrukturausfälle eher die Ausnahme darstellen, dass perspektivisch aber einerseits mit Blick auf Cyberattacken, andererseits mit Blick auf die Dynamik enger werdender Beziehungen zwischen Infrastrukturen, die Risiken zunehmen. Es ist allerdings darauf zu verweisen, dass diese Entwicklungsdynamiken keineswegs allein zu einer Erhöhung der Vulnerabilität führen. Beispielsweise führen Veränderungen im Kontext der Energiewende sowohl zu einer Zunahme der Interdependenzen und der Komplexität als auch zu neuen Lösungen im Kontext der Energiewende („Micro Grids“) und der Sektorkopplung Verkehr und Energie (Flexibilisierung), sodass (Selbst-)Steuerungspotenziale entstehen, die unter geeigneten Voraussetzungen auch Kaskadeneffekte vermeiden und zu einer Minderung der Vulnerabilität beitragen können. 
Gleichwohl ist der Zugang „Verminderung der Vulnerabilität" nicht mehr hinreichend. Maßnahmen zur Erhöhung der Resilienz der Infrastrukturen sind unabdingbar. Resilienz ist nicht (allein) die Kehrseite der Vulnerabilität, sondern resiliente (Infrastruktur-)Systeme müssen fähig sein, auch mit völlig unbekannten Ereignissen umzugehen, um nach Schockereignissen schnell ihre Dienstleistungen wieder zu erbringen. Von Gleich (in: Brandt 2017) fasst den Ansatz der Resilienz unter dem Begriff "fast egal was kommt Strategie“ zusammen. Ganz im Sinne des Verständnisses von sozio-technischen Systemen geht es mithin nicht allein um technische Fragestellungen, sondern gerade auch um die organisatorische Seite (unter anderem Organisation, Vorbereitung, Handlungskapazitäten) der Bewältigung von Extremereignissen. Brand et al. (2017) verweisen auf die erforderlichen Eigenschaften von resilienten (Infrastruktur-) Systemen, wozu sie unter anderem Widerstandsfähigkeit, Adaptivität, Innovationsfähigkeit und Improvisationsfähigkeit zählen, und Gestaltungsprinzipien wie Varietät, Redundanz, Diversität sowie Kriterien für die Netzwerkgestaltung wie Modularität, Subsidiarität oder lose optionale Kopplungen.

Die vielfältigen neuen Dynamiken in der Entwicklung der Infrastruktursysteme und deren Kopplungen ermöglichen eine Minderung der Umweltbelastungen und Erhöhungen der Ressourceneffizienz. Zugleich ist mit diesen Dynamiken verbunden, dass neue und unbekannte Konstellationen entstehen. Die Relevanz der resilienten Gestaltung der Infrastrukturen nimmt zu und mithin auch der Forschungsbedarf.

\section{Literatur}

Agentur für Erneuerbare Energien (Hrsg.) (2016): Flexibilität durch Kopplung von Strom, Wärme und Verkehr. Metaanalyse. Berlin.

Bie, Z./Lin, Y./Li, G./Li, F. (2017): Battling the Extreme: A Study on the Power System Resilience. In: Proceedings of the IEEE 105/7: 1253-1266.

Bundesnetzagentur (2007): Bericht der Bundesnetzagentur für Elektrizität,

Gas, Telekommunikation, Post und Eisenbahnen über die Systemstörung im deutschen und europäischen Verbundsystem am 4. November 2006. Bonn.

Brand, U. et al. (2017): Auf dem Weg zu Resilienten Energiesystemen! Resiliente Gestaltung der Energiesysteme am Beispiel der Transformationsoptionen „EE-Methan-System" und „Regionale Selbstversorgung“. Abschlussbericht des vom BMBF geförderten Projektes RESYSTRA.

Buldyrev, S./Parshani, R./Paul, G./Stanley, H. E./Havlin, S. (2010): Catastrophic cascade of failures in interdependent networks. In: NATURE 464: 1025-1028.

Libbe, J. (2017): Gekoppelte Infrastrukturen sind nicht nur eine technische Herausforderung. In: Difu-Berichte 2/2017: 4-3.

Rinaldi, S. M./Peerenboom, J.P./Kelly, T. K. (2001): Identifying, Understanding, and Analyzing. Critical Infrastructure Interdependencies. In: IEEE Control Systems Magazine 21/6: 11-25.

Tondel, I. A./Foros, J./Kilskar, S. K./Hokstadt, P./Jaatum M. G. (2017): Interdependencies and reliability in the combined ICT and Power system: An overview over current research. In: Applied Computing and informatics, In Press.

Quaschning, V. (2016): Sektorkopplung durch die Energiewende. Anforderungen an den Ausbau erneuerbarer Energien zum Erreichen der Pariser Klimaschutzziele unter Berücksichtigung der Sektorkopplung. Berlin, HTW-Hochschule für Technik und Wirtschaft.

\section{AUTOREN + KONTAKT}

Dr. Jens Libbe leitet am Difu den Forschungsbereich "Infrastruktur, Wirtschaft und Finanzen“.

Deutsches Institut für Urbanistik gGmbH Zimmerstraße 13-15, 10969 Berlin. Tel.: +49 30 39001-115. E-Mail: libbe@difu.de, Website: www.difu.de

Ulrich Petschow ist Leiter des Forschungsfelds Umweltökonomie und Umweltpolitik am Institut für ökologische Wirtschaftsforschung (IÖW).

Institut für ökologische Wirtschaftsforschung (IÖW) $\mathrm{GmbH}$, Potsdamer Str. 105, 10785 Berlin Tel.: +49 30 884594-0. E-Mail: ulrich.petschow@ioew.de, Website: www.ioew.de
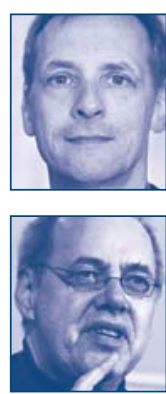

\section{Auf der SUCHE nach dem passenden GESCHENK?}

\footnotetext{
Verschenken Sie ein Jahr Ideen für eine umweltgerechte Wirtschaft
mit einem ABO von Ökologisches Wirtschaften

Vier Ausgaben für 51,- Euro statt regulär 68,- Euro (zzgl. Versand)

Bestellung an: abo@oekom.de oder+49/(089) 5441 84-25 oder unter: www.oekologisches-wirtschaften.net
}

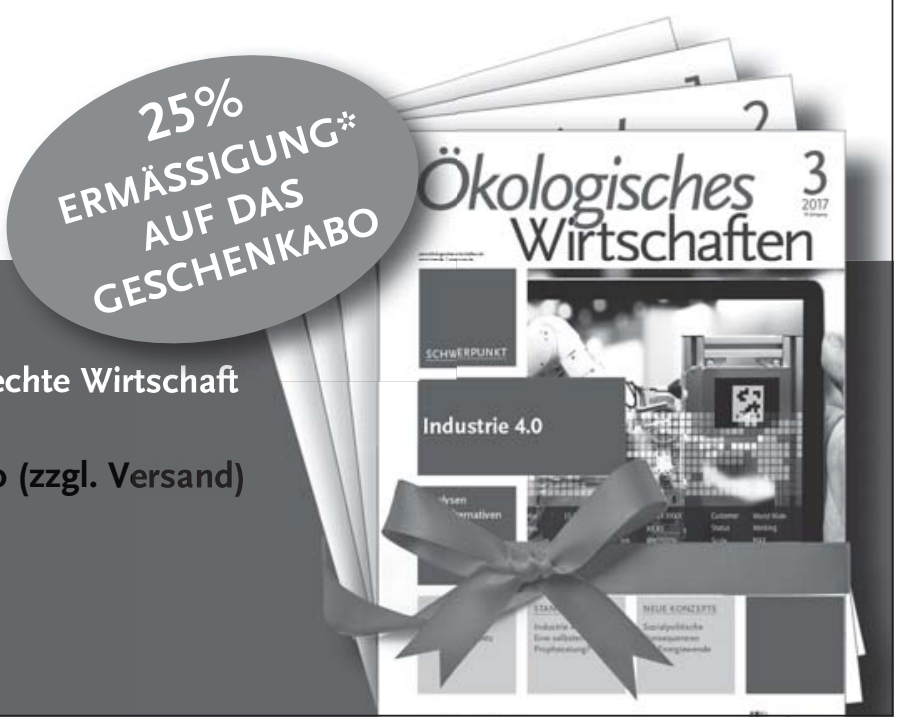

